\title{
Existence and Multiplicity Results for the Scalar Curvature Problem on the Half-Sphere $\mathbb{S}_{+}^{3}$
}

\begin{abstract}
Ridha Yacoub
Department of Mathematics and Computer Science, University of Monastir, I.P.E.I.M., Avenue Ibn Al Jazzar, 5019 Monastir, Tunisia
\end{abstract}

Correspondence should be addressed to Ridha Yacoub; ridha.yacoub@ipeim.rnu.tn

Received 3 June 2013; Accepted 27 January 2014; Published 20 March 2014

Academic Editor: Constantin Udriste

Copyright (C) 2014 Ridha Yacoub. This is an open access article distributed under the Creative Commons Attribution License, which permits unrestricted use, distribution, and reproduction in any medium, provided the original work is properly cited.

In this paper we deal with the scalar curvature problem under minimal boundary mean curvature condition on the standard 3dimensional half-sphere. Using tools related to the theory of critical points at infinity, we give existence results under perturbative and nonperturbative hypothesis, and with the help of some "Morse inequalities at infinity", we provide multiplicity results for our problem.

\section{New Results on Scalar Curvature Problem}

In this paper, we revisit a problem having a geometric origin. Namely, let

$$
\mathbb{S}_{+}^{3}=\left\{x=\left(x_{1}, x_{2}, x_{3}, x_{4}\right) \in \mathbb{R}^{4} \text {, s.t. }|x|=1, x_{4}>0\right\}
$$

be the standard 3-dimensional half-sphere endowed with its standard Riemannian metric $g$. Given a function $K: \mathbb{S}_{+}^{3} \rightarrow$ $\mathbb{R}$, we consider the problem of finding a metric $\widetilde{g}$ in the conformal class of $g$ such that $\mathscr{R}_{\tilde{g}}=K$ and $h_{\tilde{g}}=0$, where $\mathscr{R}_{\tilde{g}}$ is the scalar curvature of $\mathbb{S}_{+}^{3}$ and $h_{\tilde{g}}$ is the mean curvature of $\partial \mathbb{S}_{+}^{3}$ with respect to $\widetilde{g}$. Let $\widetilde{g}=u^{4} g$ be such a metric "conformal" to $g$; then the above problem amounts to find a smooth positive solution to the following PDE:

$$
\begin{gathered}
-\Delta_{g} u+\frac{3}{4} u=\frac{1}{8} K u^{5} \text { in } \mathbb{S}_{+}^{3}, \\
u>0 \text { in } \mathbb{S}_{+}^{3}, \\
\frac{\partial u}{\partial v}=0 \text { on } \partial \mathbb{S}_{+}^{3},
\end{gathered}
$$

where $v$ is the outward normal vector with respect to the metric $g$. Problems related to (2) were widely studied by various authors [1-12].

Note that, to solve the problem (2), the function $K$ has to be positive somewhere. Moreover, there exist topological obstructions, as Kazdan-Warner obstructions for the scalar curvature problem on $\mathbb{S}^{n}$ (see [13]). Therefore we are led to seek sufficient conditions to set on $K$, so that the problem (2) has solutions. In addition to the existence problem, we address, in this paper, the question of the number of such metrics $\widetilde{g}$ in the conformal class of $g$, with prescribed scalar curvature $K$ and zero boundary mean curvature.

In $[3,4,6,11]$, the authors have studied the problem (2). The methods of $[6,11]$ involve a fine blow-up analysis of some subcritical approximations and the use of topological degree tools. However, the methods of $[3,4]$ make use of algebraic topological and dynamical tools, coming from the theory of critical points at infinity (see [14]); we also have addressed this problem in [12], using similar tools.

The main contribution of the present work is to generalize certain previous existence results of $[3,12]$ and to give new existence results to which we add multiplicity results, using tools coming from the theory of critical points at infinity.

In the first part of this paper, we provide existence and multiplicity results under perturbative hypothesis.

In order to state our results, we introduce the following notations and assumptions.

Through the whole of this paper, we assume that $K_{1}=$ $K_{/ \partial S_{+}^{3}}$ has a finite set of nondegenerate critical points,

$$
\mathscr{K}=\left\{y \in \partial \mathbb{S}_{+}^{3} \text { s.t. } \nabla K_{1}(y)=0\right\}=\left\{y_{0}, y_{1}, \ldots, y_{\ell}\right\},
$$


ordered such that

$$
K_{1}\left(y_{0}\right) \geqslant K_{1}\left(y_{1}\right) \geqslant \cdots \geqslant K_{1}\left(y_{\ell}\right) .
$$

We define the following sets:

$$
\begin{gathered}
\mathscr{I}_{+}=\left\{y \in \mathscr{K} \text { s.t. } \frac{\partial K}{\partial \nu}(y)>0\right\}, \\
\mathscr{I}_{+}^{0}=\left\{y \in \mathscr{K} \text { s.t. } \frac{\partial K}{\partial \nu}(y)=0,-\Delta_{g} K(y)>0\right\} .
\end{gathered}
$$

Let $Z$ be a pseudogradient of $K_{1}$ of Morse-Smale type; that is, the intersections of unstable and stable manifolds of the critical points of $K_{1}$, with respect to $Z$, are transverse. For $y \in$ $\mathscr{K}$, we denote by $W_{u}(y)$ and $W_{s}(y)$, respectively, the unstable manifold of $y$ and the stable manifold of $y$ with respect to $Z$, and we denote by $\operatorname{ind}\left(K_{1}, y\right)$ the Morse index of $K_{1}$ at $y$, that is, the dimension of the submanifold $W_{u}(y)$.

We introduce the following assumptions:

$$
\left(\mathrm{A}_{0}\right) W_{u}\left(y_{j}\right) \cap W_{s}\left(y_{i}\right)=\emptyset \text { for each } y_{j} \in \mathscr{K} \backslash\left(\mathscr{I}_{+} \cup \mathscr{I}_{+}^{0}\right)
$$
and $y_{i} \in \mathscr{I}_{+} \cup \mathscr{F}_{+}^{0}$.

$\left(\mathrm{A}_{1}\right)$ For each critical point $y$ of $K_{1}$ such that $(\partial K / \partial \nu)(y)=$ 0 we have $\Delta_{g} K(y) \neq 0$; furthermore, there is a constant $\bar{r}>0$ such that $-\Delta_{g} K(y)(\partial K / \partial \nu)(a) \geqslant 0$, for all $a \in B(y, \bar{r}) \cap \partial \mathbb{S}_{+}^{3}$.

$\left(\mathrm{A}_{2}\right)$ For all $y \in \mathscr{I}_{+} \cup \mathscr{F}_{+}^{0}$, $\operatorname{ind}\left(K_{1}, y\right) \geqslant 1$.

We then have the following perturbative result.

Theorem 1. Under assumptions, $\left(A_{0}\right),\left(A_{1}\right)$, and $\left(A_{2}\right)$, there exists a constant $c_{0}>0$ independent of the function $K$ such that if

$$
\left\|K_{1}-1\right\|_{L^{\infty}\left(\partial \mathbb{S}_{+}^{3}\right)}<c_{0},
$$

and if

$$
\sum_{y \in \mathscr{F}_{+} \cup \mathcal{F}_{+}^{0}}(-1)^{2-i n d\left(K_{1}, y\right)} \neq 1
$$

then the problem (2) has at least one solution.

Further, if one assumes that all the solutions of (2) are nondegenerate, then one has

$$
\# \mathscr{C} \geqslant\left|1-\sum_{y \in \mathcal{F}_{+} \cup \mathcal{F}_{+}^{0}}(-1)^{2-i n d\left(K_{1}, y\right)}\right|,
$$

where $\# \mathscr{C}$ is the cardinality of the set of solutions of (2).

Note 1. We recall here that for a generic function, $K$, it follows from the Sard-Smale theorem that all the solutions of (2) are nondegenerate. See, for example [15], for a related discussion on this. Thereby, in the assumptions of multiplicity results, one may replace expression "Further, if we assume that all the solutions of (2) are nondegenerate, then we have..." by expression "Further, for a generic function $K$, we have...".

We recall also that $w \in \mathscr{C}$ is said to be a nondegenerate solution, if zero is not eigenvalue of the associated linearized operator $-\Delta_{g}+3 / 4-(1 / 8) K w^{4}$.
Remark 2. The existence result of Theorem 1 is slightly different from the one of Theorem 1.3 of [3], since the required assumptions by the two theorems are not all the same. Further, Theorem 1 also gives us a multiplicity result.

We will provide a more general result than Theorem 1 . For this, we define, for each index $i, 0 \leqslant i \leqslant \ell$, the set

$$
X_{i}=\bigcup_{\substack{0 \leqslant j \leqslant i \\ y_{j} \in \mathcal{F}_{+} \cup \mathscr{F}_{+}^{0}}} \overline{W_{s}}\left(y_{j}\right) .
$$

Under the assumption $\left(\mathrm{A}_{2}\right)$, we see that $X_{i} \varsubsetneqq \partial \mathbb{S}_{+}^{3}=\mathbb{S}^{2}$; hence $X_{i}$ is contractible in $\mathbb{S}^{2}$. Let us denote by $\theta:[0,1] \times X_{i} \rightarrow \mathbb{S}^{2}$ this contraction and let

$$
c_{i}=\min K \circ \theta .
$$

We now introduce the following assumptions:

$\left(\mathrm{A}_{3}\right)\left|K\left(y_{0}\right) / c_{i}-1\right|<c_{0}$, where $c_{0}$ is given in Theorem 1 .

$\left(\mathrm{A}_{4}\right)$ For all $y \in\left\{y_{i+1}, \ldots, y_{\ell}\right\} \cap \mathscr{I}_{+} \cup \mathscr{I}_{+}^{0}, K(y)<c_{i}$.

We then have the following existence and multiplicity result.

Theorem 3. Under the conditions, $\left(A_{0}\right),\left(A_{1}\right),\left(A_{2}\right),\left(A_{3}\right)$, and $\left(A_{4}\right)$, if

$$
\sum_{\substack{y_{j} \in \mathcal{F}_{\mathcal{F}} \cup \mathcal{F}_{+}^{0} \\ 0 \leqslant j \leqslant i}}(-1)^{2-i n d\left(K_{1}, y_{j}\right)} \neq 1,
$$

then the problem (2) has at least one solution.

Further, if one assumes that all the solutions of (2) are nondegenerate, then one has

$$
\# \mathscr{C} \geqslant\left|1-\sum_{\substack{y_{j} \in \mathcal{F}_{+} \cup \mathcal{F}_{+}^{0} \\ 0 \leqslant j \leqslant i}}(-1)^{2-i n d\left(K_{1}, y_{j}\right)}\right| .
$$

Remark 4. Theorem 3 is more general than Theorem 1 for two reasons. First, because, in Theorem $3, K$ is close to a constant only in a prescribed region of $\partial \mathbb{S}_{+}^{3}$ and not in all $\partial \mathbb{S}_{+}^{3}$. Secondly, the count-index formula of Theorem 3 is more general than the one of Theorem 1, since the formula of Theorem 1 is obtained for $i=\ell$ from the one of Theorem 3 .

In the second part of this work, we will establish nonperturbative results. For this, denoting by $\mathrm{d}$ the geodesic distance on $\mathbb{S}_{+}^{3}$, let $G_{1}$ be the function defined, for $x \neq y$, by

$$
G_{1}(x, y)=\frac{1}{\sqrt{1-\cos \mathrm{d}(x, y)}} .
$$

And now, denoting by $\# \mathscr{I}_{+}$the cardinality of $\mathscr{I}_{+}$, let us introduce, for any integer $p, 1 \leqslant p \leqslant \# \mathscr{I}_{+}$, the set

$$
\mathscr{F}_{p}=\left\{\left(y_{i_{1}}, \ldots, y_{i_{p}}\right) \in\left(\mathscr{I}_{+}\right)^{p}, y_{i_{j}} \neq y_{i_{k}} \text { for } j \neq k\right\} .
$$


For all $p$-tuple $\left(y_{i_{1}}, \ldots, y_{i_{p}}\right) \in \mathscr{F}_{p}$ we define the matrix $M\left(y_{i_{1}}, \ldots, y_{i_{p}}\right)=\left(m_{j k}\right)_{\substack{1 \leqslant p \leqslant j \\ 1 \leqslant k \leqslant p}}$ by

$$
\begin{aligned}
m_{j j} & =\frac{1}{K\left(y_{i_{j}}\right)^{3 / 2}} \frac{\partial K}{\partial \nu}\left(y_{i_{j}}\right), \\
m_{j k} & =-\frac{4 \sqrt{2} G_{1}\left(y_{i_{j}}, y_{i_{k}}\right)}{\left(K\left(y_{i_{j}}\right) K\left(y_{i_{k}}\right)\right)^{1 / 4}}
\end{aligned}
$$

$$
\text { for } j \neq k \text {. }
$$

Now we formulate the following assumptions:

$\left(\mathrm{A}_{5}\right) \forall\left(y_{i_{j}}, y_{i_{k}}\right) \in \mathscr{F}_{2}$; we have $m_{j j} m_{k k}<m_{j k}^{2}$.

$\left(\mathrm{A}_{6}\right) \forall$ critical point $y$ of $K_{1}$, we have $(\partial K / \partial \nu)(y) \leqslant 0$.

We then have the following.

Theorem 5. Under the conditions, $\left(A_{0}\right),\left(A_{1}\right)$, and $\left(A_{5}\right)$ or $\left(A_{6}\right)$, if there exists an index $i, 1 \leqslant i \leqslant \ell$, such that

$\left(H_{\mathrm{i}}\right)$

$$
\begin{aligned}
& \forall y \in\left(\mathscr{I}_{+} \cup \mathscr{I}_{+}^{0}\right) \cap\left\{y_{i+1}, \ldots, y_{\ell}\right\} \\
& \frac{1}{K(y)^{1 / 2}}>\frac{1}{K\left(y_{0}\right)^{1 / 2}}+\frac{1}{K\left(y_{i}\right)^{1 / 2}},
\end{aligned}
$$

and if

$$
\sum_{\substack{y_{j} \in \mathcal{F}_{+} \cup \mathcal{F}_{+}^{0} \\ 0 \leqslant j \leqslant i}}(-1)^{2-i n d\left(K_{1}, y_{j}\right)} \neq 1,
$$

then the problem (2) has at least one solution.

If in addition one assumes that all the solutions of (2) are nondegenerate, then

$$
\# \mathscr{C} \geqslant\left|1-\sum_{\substack{y_{j} \in \mathcal{I}_{+} \cup \mathcal{F}_{+}^{0} \\ 0 \leqslant j \leqslant i}}(-1)^{2-i n d\left(K_{1}, y_{j}\right)}\right| .
$$

As a corollary of Theorem 5, one has the following.

Corollary 6. Under the conditions, $\left(A_{0}\right),\left(A_{1}\right)$, and $\left(A_{5}\right)$ or $\left(A_{6}\right)$, if

$$
\sum_{y \in \mathcal{F}_{+} \cup \mathscr{F}_{+}^{0}}(-1)^{2-i n d\left(K_{1}, y\right)} \neq 1,
$$

then the problem (2) has at least one solution.

Furthermore, if we assume that all the solutions of (2) are nondegenerate, then

$$
\# \mathscr{C} \geqslant\left|1-\sum_{y \in \mathscr{F}_{+} \cup \mathscr{F}_{+}^{0}}(-1)^{2-i n d\left(K_{1}, y\right)}\right| .
$$

Remark 7. The result of Corollary 6 can be recovered by Theorem 1.1 and Corollary 1.2 in [3] and by Theorem 1.2 and Corollary 1 in [4], and it completes the existence result of Corollary 1.1 in [12] by a multiplicity result.

Commentary. We point out that the main new contribution of Theorem 5 (as well as that of Theorem 3 ) is that we address here the case where the total sum

$$
\sum_{y \in \mathscr{I}_{+} \cup \mathscr{F}_{+}^{0}}(-1)^{2-\operatorname{ind}\left(K_{1}, y\right)}
$$

is equal to 1 , but a partial sum

$$
\sum_{\substack{y_{j} \in \mathcal{I}_{+} \cup \mathcal{F}_{+}^{0} \\ 0 \leqslant j \leqslant i}}(-1)^{2-\operatorname{ind}\left(K_{1}, y_{j}\right)}
$$

is not equal to 1 . The main issue is to take advantage of such information to prove the existence of solutions to the problem (2). Notice that an interpretation of the fact that the total sum is different from one is that the topological contribution of the critical points at infinity to the topology of the level sets of the associated Euler-Lagrange functional is not trivial.

In view of such an interpretation, we raise the following question: what happens if the total contribution is trivial, but a subset of critical points at infinity induce a nontrivial difference of topology; can we still use such a topological information to prove existence of solutions?

With respect to the above question, Theorem 5 (and also Theorem 3) gives a sufficient condition to be able to derive from such local information the existence of solutions for the problem (2).

As pointed out above, our result does not only give existence results but also, under generic conditions, gives a lower bound on the number of solutions of (2). Such a result is reminiscent to the celebrated Morse Theorem, which states that the number of critical points of a Morse function defined on a compact manifold is lower-bounded in terms of the topology of the underlying manifold. Our result can be seen as a kind of Morse Inequality at Infinity. Indeed, it gives a lower bound on the number of metrics with prescribed scalar curvature and zero boundary mean curvature, in terms of the topology at infinity.

In what follows we show a situation where Corollary 6 does not work, while Theorem 5 allows having solutions to problem (2).

Example. Let $K_{1}: \mathbb{S}^{2} \rightarrow \mathbb{R}$ be such that

$$
\begin{aligned}
& \mathscr{I}_{+}^{0}=\left\{y_{0}, y_{1}, y_{2}, y_{3}\right\}, \\
& \mathscr{I}_{+}=\left\{y_{4}\right\}, \\
& \operatorname{ind}\left(K_{1}, y_{0}\right)=\operatorname{ind}\left(K_{1}, y_{3}\right)=\operatorname{ind}\left(K_{1}, y_{4}\right)=2,
\end{aligned}
$$

and

$$
\operatorname{ind}\left(K_{1}, y_{1}\right)=\operatorname{ind}\left(K_{1}, y_{2}\right)=1
$$

It is easy to compute that

$$
\sum_{y \in \mathcal{F}_{+} \cup \mathcal{F}_{+}^{0}}(-1)^{2-\operatorname{ind}\left(K_{1}, y\right)}=1,
$$


and then Corollary 6 does not work. But if we have

$$
\frac{1}{K\left(y_{3}\right)^{1 / 2}}>\frac{1}{K\left(y_{0}\right)^{1 / 2}}+\frac{1}{K\left(y_{2}\right)^{1 / 2}},
$$

using Theorem 5 with $i=2$, then we have

$$
\left|1-\sum_{\substack{y_{j} \in \mathcal{F}_{+} \cup \mathcal{F}_{+}^{0} \\ 0 \leqslant j \leqslant 2}}(-1)^{2-\operatorname{ind}\left(K_{1}, y_{j}\right)}\right|=2 \neq 1,
$$

and thus (2) has at least a solution. If solutions of (2) are assumed to be nondegenerate, we derive the existence of at least two solutions.

The rest of our paper is organized as follows. In Section 2, we set the general framework and recall some basic known facts. Section 3 is devoted to the proofs of Theorem 1 and Theorem 3. Finally, we prove Theorem 5 and Corollary 6 in Section 4 .

\section{Known Facts about Scalar Curvature Problem}

In this Section we recall the variational formulation of the problem (2), as well as some previous useful results. We introduce on $H^{1}\left(\mathbb{S}_{+}^{3}, \mathbb{R}\right)$ the norm

$$
\|u\|^{2}=\int_{\mathbb{S}_{+}^{3}}\left(-\Delta_{g} u+\frac{3}{4} u\right) u d v_{g}
$$

associated with the Yamabe operator $-\Delta_{g}+3 / 4$, where $d v_{g}$ is the volume element of $g$ on $\mathbb{S}_{+}^{3}$. Now we define $\sum=\{u \in$ $\left.H^{1},\|u\|=1\right\}$ to be the unit sphere of $H^{1}\left(\mathbb{S}_{+}^{3}, \mathbb{R}\right)$ and $\Sigma^{+}=$ $\left\{u \in \sum, u \geq 0\right\}$. The Euler functional on $H^{1}\left(\mathbb{S}_{+}^{3}, \mathbb{R}\right)$ associated with the problem (2) is

$$
J(u)=\frac{\|u\|^{2}}{\left(\int_{\mathbb{S}_{+}^{3}} K u^{6} d v_{g}\right)^{1 / 3}} .
$$

The problem (2) then amounts to find a critical point of $J$ under the constraint $u \in \sum^{+}$. The difficulty in this problem comes from the fact that the functional $J$ fails to satisfy the Palais-Smale condition on $\sum^{+}$. This failure was studied by various authors; see for example [16-18]. To characterize the sequences violating the Palais-Smale condition on $\Sigma^{+}$, we need to fix some notations. For $(a, \lambda) \in \overline{\mathbb{S}_{+}^{3}} \times(0,+\infty)$ let

$$
\delta_{(a, \lambda)}(x)=\sigma \frac{\sqrt{\lambda}}{\sqrt{\lambda^{2}+1-\left(\lambda^{2}-1\right) \cos d(a, x)}},
$$

where $d$ is the geodesic distance on $\left(\mathbb{S}_{+}^{3}, g\right)\left(\delta_{(a, \lambda)}\right.$ is known to be the solution of the Yamabe problem on the Sphere $\mathbb{S}^{3}$ ) and $\sigma$ is chosen so that

$$
-\Delta_{g} \delta_{(a, \lambda)}+\frac{3}{4} \delta_{(a, \lambda)}=\delta_{(a, \lambda)}^{5}
$$

is satisfied on $\mathbb{S}_{+}^{3}$. Observe that, if $a \in \partial \mathbb{S}_{+}^{3}$, we have $\partial \delta_{(a, \lambda)} / \partial v=0$ on $\partial \mathbb{S}_{+}^{3}$. However, $\partial \delta_{(a, \lambda)} / \partial \nu \neq 0$ if $a \notin$ $\partial \mathbb{S}_{+}^{3}$; then in this case we need to introduce another function $\varphi_{(a, \lambda)}$ which satisfies

$$
\begin{gathered}
-\Delta_{g} \varphi_{(a, \lambda)}+\frac{3}{4} \varphi_{(a, \lambda)}=\delta_{(a, \lambda)}^{5} \text { in } \mathbb{S}_{+}^{3}, \\
\frac{\partial \varphi_{(a, \lambda)}}{\partial \nu}=0 \text { on } \partial \mathbb{S}_{+}^{3} .
\end{gathered}
$$

We will write in the sequel $\delta_{i}$ for $\delta_{\left(a_{i}, \lambda_{i}\right)}$ and $\varphi_{i}$ for $\varphi_{\left(a_{i}, \lambda_{i}\right)}$.

Let $w$ be a nondegenerate solution of (2) or zero. Then, for $\epsilon>0$ and integers $p, q$ such that $1 \leqslant q+p$, we define

$$
\begin{aligned}
& V(p, q, \epsilon, w)\left\{u \in \sum^{+} / \exists a_{1}, \ldots, a_{p+q} \in \overline{\mathbb{S}_{+}^{3}},\right. \\
& \exists \lambda_{1}, \ldots, \lambda_{p+q}>\frac{1}{\epsilon}, \\
& \exists \alpha_{0}, \ldots, \alpha_{p+q}>0, \\
& \text { s.t. }\left|\alpha_{0}^{4} J(u)^{3}-1\right|<\epsilon, \\
& \| u-\alpha_{0} w-\sum_{i=1}^{p} \alpha_{i} \delta_{i}-\sum_{i=p+1}^{p+q} \alpha_{i} \varphi_{i} \mid<\epsilon, \\
& \varepsilon_{i j}<\epsilon,\left|\frac{\alpha_{i}^{4} K\left(a_{i}\right)}{\alpha_{j}^{4} K\left(a_{j}\right)}-1\right|<\epsilon \\
& \text { for } 1 \leqslant i \neq j \leqslant p+q, \\
& \lambda_{i} d_{i}<\epsilon \text { for } 1 \leqslant i \leqslant p, \\
&\left.\lambda_{i} d_{i}>\frac{1}{\epsilon} \text { for } p+1 \leqslant i \leqslant p+q\right\},
\end{aligned}
$$

where $d_{i}=\mathrm{d}\left(a_{i}, \partial \mathbb{S}_{+}^{3}\right)$ and $\varepsilon_{i j}=\left(\lambda_{i} / \lambda_{j}+\lambda_{j} / \lambda_{i}+\lambda_{i} \lambda_{j}(1-\right.$ $\left.\left.\cos \mathrm{d}\left(a_{i}, a_{j}\right)\right)\right)^{-1 / 2}$. Note that, when $w=0$, to write the definition of $V(p, q, \epsilon, 0)$, we replace $w$ by 0 and we remove $\alpha_{0}$ and the condition $\left|\alpha_{0}^{4} J(u)^{3}-1\right|<\epsilon$ in the definition of $V(p, q, \epsilon, w)$ above.

The failure of the Palais-Smale condition can be described (see Proposition 1 in [4]), as follows.

Proposition 8. Let $\left(u_{k}\right)$ be a sequence in $\sum^{+}$such that $J\left(u_{k}\right)$ is bounded and $\partial J\left(u_{k}\right) \rightarrow 0$. Then, there exist integers $p, q$ such that $1 \leqslant q+p$, a sequence $\epsilon_{k}>0\left(\epsilon_{k} \rightarrow 0\right)$, and an extracted subsequence of $\left(u_{k}\right)$, again denoted by $\left(u_{k}\right)$, such that $\forall k \in \mathbb{N}, u_{k} \in V\left(p, q, \epsilon_{k}, w\right)$, where $w$ is either solution of (2) or zero.

Here $\partial J$ is the gradient of $J$ with respect of the $H^{1}$-inner product:

$$
(u, v)=\int_{\mathbb{S}_{+}^{3}}\left(-\Delta_{g} u+\frac{3}{4} u\right) v d v_{g} .
$$


We consider the following minimization problem for a function $u$ belonging to $V(p, q, \epsilon, w)$, with $\epsilon$ small:

$$
\begin{gathered}
\min \left\|u-\alpha_{0}(w+h)-\sum_{i=1}^{p} \alpha_{i} \delta_{i}-\sum_{i=p+1}^{p+q} \alpha_{i} \varphi_{i}\right\|, \\
\text { for } \begin{cases}\alpha_{0}, \alpha_{i}, \lambda_{i}>0, & 1 \leqslant i \leqslant p+q \\
a_{i} \in \partial \mathbb{S}_{+}^{3}, & 1 \leqslant i \leqslant p \\
a_{i} \in \mathbb{S}_{+}^{3}, & p+1 \leqslant i \leqslant p+q \\
h \in T_{w}\left(W_{u}(w)\right), & \end{cases}
\end{gathered}
$$

where $T_{w}\left(W_{u}(w)\right)$ is the tangent space at $w$ to $W_{u}(w)$ the unstable manifold of $w$ for a pseudogradient of $J$. We then have the next proposition which defines a parameterization of the set $V(p, q, \epsilon, w)$. It follows from the corresponding statement in [19] (see also Proposition 4 in [4]).

Proposition 9. For any integers $p, q$, such that $1 \leqslant q+p$, there exists $\epsilon(p, q)>0$ such that if $\epsilon<\epsilon(p, q)$ and $u \in V(p, q, \epsilon, w)$, the minimization problem (33) has a unique solution (up to permutation). follows:

Thus, we can write any $u \in V(p, q, \epsilon, w)$ uniquely as

$$
u=\sum_{i=1}^{p} \bar{\alpha}_{i} \delta_{\left(\bar{a}_{i}, \bar{\lambda}_{i}\right)}+\sum_{i=p+1}^{p+q} \bar{\alpha}_{i} \varphi_{\left(\bar{a}_{i}, \bar{\lambda}_{i}\right)}+v+\bar{\alpha}_{0}(w+\bar{h}),
$$

where $\left(\bar{\alpha}_{0}, \bar{\alpha}_{1}, \ldots, \bar{\alpha}_{p+q}, \bar{a}_{1}, \ldots, \bar{a}_{p+q}, \bar{\lambda}_{1}, \ldots, \bar{\lambda}_{p+q}, \bar{h}\right)$ is the solution of the minimization problem (33) and $v \in H^{1}\left(\mathbb{S}_{+}^{3}\right) \cap$ $T_{w}\left(W_{s}(w)\right)$ is as follows:

$$
\begin{gathered}
(v, \psi)=0 \text { for } \psi \in\left\{\delta_{i}, \frac{\partial \delta_{i}}{\partial a_{i}}, \frac{\partial \delta_{i}}{\partial \lambda_{i}}\right\}_{i=1}^{i=p} \bigcup\left\{\varphi_{i}, \frac{\partial \varphi_{i}}{\partial a_{i}}, \frac{\partial \varphi_{i}}{\partial \lambda_{i}}\right\}_{i=p+1}^{i=p+q} \\
(v, w)=0, \quad(v, h)=0 \quad \forall h \in T_{w}\left(W_{u}(w)\right),\|v\|<\epsilon,
\end{gathered}
$$

where $\delta_{i}=\delta_{\left(a_{i}, \lambda_{i}\right)}, \varphi_{i}=\varphi_{\left(a_{i}, \lambda_{j}\right)}$, and $T_{w}\left(W_{s}(w)\right)$ is the tangent space at $w$ to $W_{s}(w)$ the stable manifold of $w$ for a pseudogradient of $J$.

We also consider the case, where $w=0$; then the condition (35) becomes

$$
(\nu, \psi)=0 \text { for } \psi \in\left\{\delta_{i}, \frac{\partial \delta_{i}}{\partial a_{i}}, \frac{\partial \delta_{i}}{\partial \lambda_{i}}\right\}_{i=1}^{i=p} \bigcup\left\{\varphi_{i}, \frac{\partial \varphi_{i}}{\partial a_{i}}, \frac{\partial \varphi_{i}}{\partial \lambda_{i}}\right\}_{i=p+1}^{i=p+q},
$$

From similar statements in $[14,20,21]$ (see also Proposition 2.2 in [3] or Proposition 3 in [4]), we have then the following.

Proposition 10. There exists a $\mathscr{C}^{1}$ map which, to each

$$
(\alpha, a, \lambda)=\left(\alpha_{1}, \ldots, \alpha_{p+q}, a_{1}, \ldots, a_{p+q}, \lambda_{1}, \ldots, \lambda_{p+q}\right)
$$

such that $\sum_{i=1}^{p} \alpha_{i} \delta_{i}+\sum_{i=p+1}^{p+q} \alpha_{i} \varphi_{i}+v \in V(p, q, \epsilon, 0)$, with $\epsilon$ small, associates $\bar{v}=\bar{v}(\alpha, a, \lambda)$, satisfying

$$
\begin{aligned}
& J\left(\sum_{i=1}^{p} \alpha_{i} \delta_{i}+\sum_{i=p+1}^{p+q} \alpha_{i} \varphi_{i}+\bar{v}\right) \\
& =\min _{\text {vverifying(36) }} J\left(\sum_{i=1}^{p} \alpha_{i} \delta_{i}+\sum_{i=p+1}^{p+q} \alpha_{i} \varphi_{i}+v\right) .
\end{aligned}
$$

Moreover, there exists $c>0$ such that

$$
\begin{gathered}
\|\bar{v}\| \leqslant c\left(\sum_{i=1}^{p} \frac{\left|\nabla K\left(a_{i}\right)\right|}{\lambda_{i}}+\frac{1}{\lambda_{i}^{2}}+\sum_{j=p+1}^{p+q} \frac{1}{\lambda_{j}^{2} d_{j}^{2}}\right. \\
\left.+\sum_{k \neq l} \varepsilon_{k l}\left(\ln \varepsilon_{k l}^{-1}\right)^{1 / 3}\right) .
\end{gathered}
$$

The next proposition characterizes the critical points at infinity of the associated variational problem. We recall that critical points at infinity are the orbits of the gradient flow of $J$ which remain in $V(p, q, \epsilon(s), w)$, where $\epsilon(s)$ is a function tending to 0 when $s \rightarrow+\infty$ (see [14]).

Proposition 11. Assume that, for any $\left(y_{i_{1}}, \ldots, y_{i_{p}}\right) \in \mathscr{F}_{p}$, $p \geqslant 1$, the matrix $M\left(y_{i_{1}}, \ldots, y_{i_{p}}\right)$ is nondegenerate. Under the assumption, $\left(A_{1}\right)$, the critical points at infinity of $J$ are in $V(p, 0, \epsilon, 0)$. More precisely consider the following. If $p=1$, they correspond to single bubbles $\delta_{(y,+\infty)}, y \in\left(\mathscr{I}_{+}^{0} \cup \mathscr{I}_{+}\right)$. The Morse index of $J$ at its critical point at infinity $\delta_{(y,+\infty)}$ is

$$
\operatorname{ind}\left(J, \delta_{(y,+\infty)}\right)=2-\operatorname{ind}\left(K_{1}, y\right)
$$

where ind $\left(K_{1}, y\right)$ is the Morse index of $K_{1}$ at its critical point $y$.

(1) If $p \geqslant 2$, they correspond to combinations $\sum_{j=1}^{p}\left(1 / K\left(y_{i_{j}}\right)^{4}\right) \delta_{\left(y_{i_{j}},+\infty\right)}$, where $\left(y_{i_{1}}, \ldots, y_{i_{p}}\right) \in \mathscr{F}_{p}$ is such that $\rho\left(y_{i_{1}}, \ldots y_{i_{p}}\right)>0$, where $\rho\left(y_{i_{1}}, \ldots y_{i_{p}}\right)$ is the smallest eigenvalue of the matrix $M\left(y_{i_{1}}, \ldots, y_{i_{p}}\right)$.

Proof. Using Corollary 3 of [4], there are no critical points at infinity for $J$ in $V(p, q, \epsilon, w)$ with $w \neq 0$. Hence they are in $V(p, q, \epsilon, 0)$. Using Corollary 5 of [4], there are no critical points at infinity for $J$ in $V(0, q, \epsilon, w)$ with $q \neq 0$; that is, there are no bubbles (or blow-ups) with all concentration points interior to $\mathbb{S}_{+}^{3}$. Then, using Proposition 3.1 of [3], we rule out the existence of critical points at infinity in $V(p, q, \epsilon, w)$ with $p q \neq 0$ (the so-called mixed bubbles), which signifies that bubbles concentrate uniquely in points of the boundary $\partial \mathbb{S}_{+}^{3}$; that is to say, critical points at infinity are in $V(p, 0, \epsilon, 0), p \geqslant$ 1. Finally, using Corollary 6 of [4], we derive the result.

\section{Proofs of Theorems 1 and 3}

We start the proofs by recalling the following results. 
Lemma 12. For $u=\alpha \delta_{(a, \lambda)} \in V(1,0, \epsilon, 0)$ such that $a \in B(y)$ a small enough neighborhood of $y \in \mathscr{J}_{+}^{0} \cup \mathscr{I}_{+}$a critical point of $K_{1}$, there is a change of variables $(\widetilde{a}, \widetilde{\lambda})$ such that if $y \in \mathscr{I}_{+}$,

$$
\begin{aligned}
J(u) & =\psi_{1}(\tilde{a}, \tilde{\lambda}) \\
& =\left(\frac{S_{3}}{2}\right)^{2 / 3} \frac{1}{K(\widetilde{a})^{1 / 3}}\left(1+\frac{c}{\tilde{\lambda}} \frac{\partial K}{\partial \nu}(y)+o\left(\frac{1}{\tilde{\lambda}}\right)\right),
\end{aligned}
$$

and if $y \in \mathscr{F}_{+}^{0}$, after another change of variable $\widetilde{\lambda} \mapsto \tilde{\tilde{\lambda}}$,

$$
\begin{aligned}
J(u) & =\psi_{2}(\tilde{a}, \tilde{\tilde{\lambda}}) \\
& =\left(\frac{S_{3}}{2}\right)^{2 / 3} \frac{1}{K(\widetilde{a})^{1 / 3}}\left(1-\tilde{c} \frac{\Delta_{g} K(y)}{\widetilde{\tilde{\lambda}}^{2}}+o\left(\frac{1}{\widetilde{\tilde{\lambda}}^{2}}\right)\right),
\end{aligned}
$$

where $c$ and $\tilde{c}$ are positive constants and $S_{3}$ is the Sobolev constant for $\mathbb{S}^{3}$. (We recall that $\lambda, \tilde{\lambda}$ and $\tilde{\tilde{\lambda}}$ are of the same order of size).

Lemma 12 is easily deduced from Proposition 2.8 in [3].

Lemma 13 (see [3], Lemma 4.1). Let $a_{1}, a_{2} \in \partial \mathbb{S}_{+}^{3}$, and $\alpha_{1}, \alpha_{2}>0$. For $\lambda$ large enough positive parameter, let $u=$ $\alpha_{1} \delta_{\left(a_{1}, \lambda\right)}+\alpha_{2} \delta_{\left(a_{2}, \lambda\right)}$. Then

$$
\begin{aligned}
J(u) & \leqslant\left(\frac{S_{3}}{2}\right)^{2 / 3}\left(\frac{1}{K\left(a_{1}\right)^{1 / 2}}+\frac{1}{K\left(a_{2}\right)^{1 / 2}}\right)^{2 / 3}(1+o(1)) \\
& =c_{\infty}\left(a_{1}, a_{2}\right)(1+o(1)) .
\end{aligned}
$$

Lemma 14. For $\epsilon>0$ small enough and $u=\left(\sum_{i=1}^{p} \alpha_{i} \delta_{\left(a_{i}, \lambda_{i}\right)}+\right.$ $v) \in V(p, 0, \epsilon, 0)$, one has the expansion

$$
J(u)=\left(\frac{S_{3}}{2}\right)^{2 / 3} \frac{\sum_{i=1}^{p} \alpha_{i}^{2}}{\left(\sum_{i=1}^{p} \alpha_{i}^{6} K\left(a_{i}\right)\right)^{1 / 3}}(1+o(1)) .
$$

Lemma 14 is a particular case (for $n=1$ ) of Proposition 2.4 of [3].

Proof of Theorem 1. Let

$$
c_{1}=\frac{3}{2}\left(\frac{S_{3}}{2}\right)^{2 / 3} .
$$

Using the expansion of $J$ provided by Lemma 14, there exists $c_{0}>0$ independent of $K_{1}$ such that if $\left\|K_{1}-1\right\|_{L^{\infty}\left(\partial S_{+}^{3}\right)}<c_{0}$, then for all $u \in V(1,0, \epsilon, 0), J(u)<c_{1}$ and $\forall u \in V(p, 0, \epsilon, 0)$ with $p \geqslant 2, J(u)>c_{1}$. Using Proposition 11, the only critical points at infinity of $J$ under the level $c_{1}$ correspond to single bubbles $\delta_{(y,+\infty)}$, where $y \in\left(\mathscr{I}_{+}^{0} \cup \mathscr{I}_{+}\right)$. We let in the sequel $\delta_{(y,+\infty)}=y_{\infty}$. Now define

$$
X=\bigcup_{y \in\left(\mathcal{F}_{+}^{0} \cup \mathcal{F}_{+}\right)} \overline{F_{s}}(y)
$$

where $W_{s}(y)$ is the stable manifold of $y$ for a decreasing pseudogradient $Z$ of the function $K_{1}$. By hypothesis $\left(\mathrm{A}_{2}\right)$, we have $X \varsubsetneqq \partial \mathbb{S}_{+}^{3}=\mathbb{S}^{2}$. Thus, $X$ is contractible in $\mathbb{S}^{2}$. We denote by $h:[0,1] \times X \rightarrow \mathbb{S}^{2}$ the associated contraction. Let

$$
X_{\infty}=\bigcup_{y \in\left(\mathcal{F}_{+}^{0} \cup \mathcal{F}_{+}\right)} \overline{W_{u}}\left(y_{\infty}\right),
$$

where $W_{u}\left(y_{\infty}\right)$ is the unstable manifolds at infinity, for the pseudogradient $V$ provided by Proposition 3.1 in [3] (or the pseudogradient $W$ provided by Proposition 9 in [4]), of the critical point at infinity $y_{\infty}$. It can be described, using the expansion of $J(u)$ given by Lemma 12 , as the product of $W_{s}(y)$, for the pseudogradient $Z$ of $K_{1}$, by $[A,+\infty)$ which is the domain of the variable $\lambda$, for some large enough positive real $A$.

The contraction $h$ gives rise to a contraction

$$
\begin{gathered}
\tilde{h}:[0,1] \times X \times[A,+\infty) \longrightarrow \Sigma^{+} \\
(t, x, \lambda) \longmapsto \frac{\delta_{(h(t, x), \lambda)}}{\left\|\delta_{(h(t, x), \lambda)}\right\|}
\end{gathered}
$$

Using Lemma $12, \forall(t, x, \lambda) \in[0,1] \times X \times[A,+\infty)$, one has

$$
\begin{aligned}
& J(\widetilde{h}(t, x, \lambda)) \\
& \quad=\left(\frac{S_{3}}{2}\right)^{2 / 3} \frac{1}{K(h(t, x))^{1 / 3}}\left(1+O\left(\frac{1}{A}\right)\right) .
\end{aligned}
$$

Since $K$ is close to 1 , we derive that $J(\widetilde{h}(t, x, \lambda))<c_{1}$, for $A$ large enough. Denoting by $R(\widetilde{h})$ the range of $\widetilde{h}$, that is, $R(\widetilde{h}):=$ $\widetilde{h}\left([0,1] \times X_{\infty}\right)$, then we derive that $R(\widetilde{h})$ is below the level $c_{1}$; that is to say, the contraction $\widetilde{h}$ is performed under the level $c_{1}$ and thus (see [22], Sections 7 and 8)

$$
R(\widetilde{h}) \simeq \bigcup_{\mathcal{J}_{+}^{0} \cup \mathcal{F}_{+}} W_{u}\left(y_{\infty}\right) \bigcup_{\substack{w \in \mathscr{C} \\ R(\tilde{h}) \cap W_{s}(w) \neq \emptyset}} W_{u}(w),
$$

where $\mathscr{C}$ is the set of critical points of $J$ or, equivalently, of the solutions of (2). Now, denoting by $\chi$ the Euler-Poincaré characteristic, and using the fact that $R(\widetilde{h})$ is a contractible set, it holds that

$$
\begin{aligned}
& 1=\chi(R(\widetilde{h}))=\sum_{y \in \mathcal{F}_{+}^{0} \cup \mathcal{F}_{+}}(-1)^{\operatorname{ind}\left(J, y_{\infty}\right)} \\
& +\sum_{\substack{w \in \mathscr{C} \\
R(\widetilde{h}) \cap W_{s}(w) \neq \emptyset}}(-1)^{\operatorname{ind}(J, w)},
\end{aligned}
$$

where $\operatorname{ind}(J, w)$ (resp., ind $\left.\left(J, y_{\infty}\right)\right)$ denotes the Morse index of $J$ at $w$ (resp., $\left.y_{\infty}\right)$. Since ind $\left(J, y_{\infty}\right)=2-\operatorname{ind}\left(K_{1}, y\right)$ 
and since $\sum_{y \in \mathscr{F}_{+}^{0} \cup \mathscr{I}_{+}}(-1)^{2-\mathrm{ind}\left(K_{1}, y\right)} \neq 1$, we derive that $\mathscr{C} \neq \emptyset$. Hence

$$
\begin{aligned}
& \left|1-\sum_{y \in \mathcal{F}_{+}^{0} \cup \mathscr{I}_{+}}(-1)^{2-\operatorname{ind}\left(K_{1}, y\right)}\right| \\
& \quad \leqslant\left|\sum_{\substack{w \in \mathscr{C} \\
R(\tilde{h}) \cap W_{s}(w) \neq \emptyset}}(-1)^{\operatorname{ind}(J, w)}\right| \leqslant \# \mathscr{C},
\end{aligned}
$$

where $\# \mathscr{C}$ is the cardinality of $\mathscr{C}$ q.e.d.

Proof of Theorem 3. Using the same arguments of the proof of Theorem 1, let

$$
X_{i \infty}=\bigcup_{\substack{0 \leqslant j \leqslant i \\ y_{j} \in \mathscr{F}_{+} \cup \mathscr{F}_{+}^{0}}} \overline{W_{s}}\left(y_{j \infty}\right),
$$

where $X_{i}$ is defined by (9). As we remarked it above, $X_{i \infty}$ can be parameterized by $X_{i} \times[A,+\infty)$. Let

$$
c_{i}^{\infty}=\left(\frac{S_{3}}{2}\right)^{2 / 3} \frac{1}{c_{i}^{1 / 3}},
$$

where $c_{i}=\min K \circ \theta$ and $\theta$ is the contraction

$$
\begin{gathered}
\theta:[0,1] \times X_{i} \longrightarrow \mathbb{S}^{2}, \\
(t, x) \longmapsto \theta(t, x)
\end{gathered}
$$

that is, for all $x \in X_{i}, \theta(0, x)=x$ and $\theta(1, x)=a_{0}$ a fixed point in $X_{i}$.

We now claim that $X_{i \infty}$ is contractible in $J_{c_{i}^{\infty}+\epsilon}$. Indeed, the contraction $\theta$ induces the following contraction:

$$
\begin{gathered}
\tilde{\theta}:[0,1] \times X_{i} \times[A,+\infty) \longrightarrow \Sigma^{+} \\
(t, x, \lambda) \longmapsto \frac{\delta_{(\theta(t, x), \lambda)}}{\left\|\delta_{(\theta(t, x), \lambda)}\right\|} .
\end{gathered}
$$

Thus, by Lemma 12, we derive, for $\lambda$ large enough,

$$
J\left(\delta_{(\theta(t, x), \lambda)}\right)=\left(\frac{S_{3}}{2}\right)^{2 / 3} \frac{1}{K(\theta(t, x))^{1 / 3}}\left(1+O\left(\frac{1}{\lambda}\right)\right) .
$$

Since $\forall t \in[0,1]$ and $\forall x \in X_{i}$, we have $K(\theta(t, x)) \geqslant c_{i}$; then, for $\lambda \geqslant A$ large enough,

$$
J\left(\delta_{(\theta(t, x), \lambda)}\right) \leqslant\left(\frac{S_{3}}{2}\right)^{2 / 3} \frac{1}{c_{i}^{1 / 3}}\left(1+O\left(\frac{1}{A}\right)\right) \leqslant c_{i}^{\infty}+\epsilon
$$

and thus $X_{i \infty}$ is contractible in $J_{c_{i}^{\infty}+\epsilon}$. Our claim follows.

On the other side, using Proposition 11, under the assumptions, $\left(\mathrm{A}_{3}\right)$ and $\left(\mathrm{A}_{4}\right)$, the critical points at infinity under the level $c_{i}^{\infty}+\epsilon$ are $y_{j \infty}$, where $y_{j} \in\left(\mathscr{I}_{+}^{0} \cup \mathscr{I}_{+}\right)$and
$0 \leqslant j \leqslant i$. Thus, denoting by $R(\widetilde{\theta})$ the range of $\widetilde{\theta}$, that is, $R(\widetilde{\theta}):=\widetilde{\theta}\left([0,1] \times X_{i \infty}\right)$, we derive that

$$
R(\tilde{\theta}) \simeq \bigcup_{\substack{0 \leqslant j \leqslant i \\ y_{j} \in \mathcal{F}_{+}^{0} \cup \mathscr{I}_{+}}} W_{u}\left(y_{j \infty}\right) \bigcup_{\substack{w \in \mathscr{C} \\ R(\tilde{\theta}) \cap W_{s}(w) \neq \emptyset}} W_{u}(w),
$$

where $\mathscr{C}$ is the set of the critical points of $J$. Our claim thus implies that

$$
\begin{aligned}
1=\chi(R(\widetilde{\theta}))= & \sum_{\substack{0 \leqslant j \leqslant i \\
y_{j} \in \mathcal{F}_{+}^{0} \cup \mathscr{I}_{+}}}(-1)^{\operatorname{ind}\left(J, y_{j \infty}\right)} \\
+ & \sum_{\substack{w \in \mathscr{C} \\
R(\tilde{\theta}) \cap W_{s}(w) \neq \emptyset}}(-1)^{\operatorname{ind}(J, w)} .
\end{aligned}
$$

Hence, arguing as above, we derive that $\mathscr{C} \neq \emptyset$, and that

$$
\begin{aligned}
& \left|1-\sum_{\substack{0 \leqslant j \leqslant i \\
y_{j} \in \mathcal{J}_{+}^{0} \cup \mathscr{I}_{+}}}(-1)^{2-\operatorname{ind}\left(K_{1}, y_{j}\right)}\right| \\
& \quad \leqslant\left|\sum_{\substack{w \in \mathscr{C} \\
R(\tilde{\theta}) \cap W_{s}(w) \neq \emptyset}}(-1)^{\operatorname{ind}(J, w)}\right| \leqslant \# \mathscr{C} .
\end{aligned}
$$

Our theorem is thereby proved.

\section{Proofs of Theorem 5 and Corollary 6.}

Proof of Theorem 5. Let

$$
c_{\infty}\left(y_{0}, y_{i}\right)=\left(\frac{S_{3}}{2}\right)^{2 / 3}\left(\frac{1}{K\left(y_{0}\right)^{1 / 2}}+\frac{1}{K\left(y_{i}\right)^{1 / 2}}\right)^{2 / 3} .
$$

Using Proposition 11, we observe that under the assumptions, $\left(A_{1}\right)$ and $\left(A_{5}\right)$ or $\left(A_{6}\right)$, there are no critical points at infinity of multiple masses, that is, in $V(p, 0, \epsilon, 0)$ for $p \geqslant 2$. Indeed, on one hand, under $\left(\mathrm{A}_{5}\right)$, we derive that, for any $p \geqslant 2$ and for any $\left(y_{i_{1}}, \ldots, y_{i_{p}}\right) \in \mathscr{F}_{p}$, the matrix $M\left(y_{i_{1}}, \ldots, y_{i_{p}}\right)$ is definite negative, and then its smallest eigenvalue $\rho\left(y_{i_{1}}, \ldots, y_{i_{p}}\right)$ is $<0$. On the other hand, under $\left(\mathrm{A}_{6}\right), \mathscr{I}_{+}=\emptyset$ and therefore $\mathscr{F}_{p}=\emptyset$ for all $p$.

Thus the critical points at infinity of our variational problem lie in $V(1,0, \epsilon, 0)$. Using the assumption $\left(\mathrm{H}_{\mathrm{i}}\right)$ of Theorem 5, it follows that the only critical points at infinity of $J$ under the level

$$
c_{i}=c_{\infty}\left(y_{0}, y_{i}\right)+\epsilon
$$

for $\epsilon$ small enough are $\delta_{\left(y_{j},+\infty\right)}=y_{j \infty}$, where $y_{j} \in\left(\mathscr{I}_{+}^{0} \cup \mathscr{I}_{+}\right)$, for $0 \leqslant j \leqslant i$.

The unstable manifolds at infinity, for the pseudogradient $V$ (provided by Proposition 3.1 in [3]), of such critical points at infinity, which we denote by $W_{u}\left(y_{j \infty}\right)$, can be described as 
the product of $W_{s}\left(y_{j}\right)$, for the pseudogradient $Z$ of $K_{1}$, by $[A,+\infty)$, domain of the variable $\lambda$, for some large enough positive real $A$. Let

$$
X_{i \infty}=\bigcup_{\substack{0 \leqslant j \leqslant i \\ y_{j} \in\left(\mathscr{F}_{+}^{0} \cup \mathscr{I}_{+}\right)}} \overline{W_{u}}\left(y_{j \infty}\right) .
$$

The set $X_{i \infty}$ can be parameterized by $X_{i} \times[A,+\infty)$, where $X_{i}$ is given by (9). We now claim that $X_{i \infty}$ is contractible in $J_{c_{i}}$, where

$$
J_{c_{i}}=\left\{u \in \Sigma^{+} \text {s.t. } J(u) \leqslant c_{i}\right\}
$$

Indeed, let $a_{1}, a_{2} \in \partial \mathbb{S}_{+}^{3}, \alpha_{1}, \alpha_{2}>0$, and $\lambda$ large enough. For $u=\alpha_{1} \delta_{\left(a_{1}, \lambda\right)}+\alpha_{2} \delta_{\left(a_{2}, \lambda\right)}$, we have, by Lemma 13, the following estimate:

$$
\begin{aligned}
J(u) & \leqslant\left(\frac{S_{3}}{2}\right)^{2 / 3}\left(\frac{1}{K\left(a_{1}\right)^{1 / 2}}+\frac{1}{K\left(a_{2}\right)^{1 / 2}}\right)^{2 / 3}(1+o(1)) \\
& =c_{\infty}\left(a_{1}, a_{2}\right)+o(1) .
\end{aligned}
$$

Let

$$
\begin{gathered}
\psi:[0,1] \times X_{i} \times[A,+\infty) \longrightarrow \Sigma^{+} \\
(t, x, \lambda) \longmapsto \frac{t \delta_{\left(y_{0}, \lambda\right)}+(1-t) \delta_{(x, \lambda)}}{\left\|t \delta_{\left(y_{0}, \lambda\right)}+(1-t) \delta_{(x, \lambda)}\right\|}
\end{gathered}
$$

The function $\psi$ is continuous, and it satisfies $\psi(0, x, \lambda)=$ $\delta_{(x, \lambda)} /\left\|\delta_{(x, \lambda)}\right\|, \quad \psi(1, x, \lambda)=\delta_{\left(y_{0}, \lambda\right)} /\left\|\delta_{\left(y_{0}, \lambda\right)}\right\|$. Now, since $K_{1}(x) \geqslant K_{1}\left(y_{i}\right)$, for any $x \in X_{i}$, it follows from inequality (66) that $J(\psi(t, x, \lambda))<c_{i}$ for all $(t, x, \lambda) \in[0,1] \times X_{i} \times$ $[A,+\infty)$. Thus, the contraction $\psi$ is performed under the level $c_{i}$. We derive that $X_{i \infty}$ is contractible in $J_{c_{i}}$, and our claim follows. On the other hand, denoting by $R(\psi)$ the range of $\psi$, that is, $R(\psi):=\psi\left([0,1] \times X_{i \infty}\right)$, we have

$$
R(\psi) \simeq \bigcup_{\substack{0 \leqslant j \leqslant i \\ y_{j} \in \mathcal{F}_{+}^{0} \cup \mathscr{I}_{+}}} W_{u}\left(y_{j \infty}\right) \bigcup_{\substack{w \in \mathscr{C} \\ R(\psi) \cap W_{s}(w) \neq \emptyset}} W_{u}(w),
$$

where $\mathscr{C}$ is the set of the critical points of $J$, and since $R(\psi)$ is contractible, it yields

$$
\begin{aligned}
1=\chi(R(\psi))= & \sum_{\substack{0 \leqslant j \leqslant i \\
y_{j} \in \mathcal{F}_{+}^{0} \cup \mathscr{F}_{+}}}(-1)^{\operatorname{ind}\left(J, y_{j \infty}\right)} \\
+ & \sum_{\substack{w \in \mathscr{C} \\
R(\psi) \cap W_{s}(w) \neq \emptyset}}(-1)^{\operatorname{ind}(J, w)} .
\end{aligned}
$$

Hence, we derive as above that $\mathscr{C} \neq \emptyset$ and that

$$
\begin{aligned}
& \left|1-\sum_{\substack{0 \leqslant j \leqslant i \\
y_{j} \in \mathcal{I}_{+}^{0} \cup \mathscr{I}_{+}}}(-1)^{2-\operatorname{ind}\left(K_{1}, y_{j}\right)}\right| \\
& \quad \leqslant\left|\sum_{\substack{w \in \mathscr{C} \\
R(\psi) \cap W_{s}(w) \neq \emptyset}}(-1)^{\operatorname{ind}(J, w)}\right| \leqslant \# \mathscr{C},
\end{aligned}
$$

where $\# \mathscr{C}$ is the number of solutions of (2). Theorem 5 follows.

Proof of Corollary 6. Recall that $K_{1}$ has only nondegenerate critical points $y_{0}, y_{1}, \ldots, y_{\ell}$, such that $K_{1}\left(y_{0}\right) \geqslant \cdots \geqslant$ $K_{1}\left(y_{\ell}\right)$. Observe that the assumption $\left(\mathrm{H}_{\mathrm{i}}\right)$ of Theorem 5 serves uniquely to exclude points $y_{j}$ of $\mathscr{I}_{+}^{0} \cup \mathscr{I}_{+}$such that $i+1 \leqslant j \leqslant \ell$, from the count-index formula. So, for the index $i=\ell$, the assumption $\left(\mathrm{H}_{\ell}\right)$ vanishes since all the critical points of $\mathscr{I}_{+}^{0} \cup \mathscr{I}_{+}$are here taken into consideration. Thus, our Corollary follows from Theorem 5 , taking $i=\ell$.

\section{Conflict of Interests}

The authors declare that there is no conflict of interests regarding the publication of this paper.

\section{References}

[1] M. Ben Ayed, K. El Mehdi, and M. Ould Ahmedou, "The scalar curvature problem on the four dimensional half sphere," Calculus of Variations and Partial Differential Equations, vol. 22, no. 4, pp. 465-482, 2005.

[2] M. Ben Ayed and H. Chtioui, "Topological tools in prescribing the scalar curvature on the half sphere," Advanced Nonlinear Studies, vol. 4, no. 2, pp. 121-148, 2004.

[3] M. Ben Ayed and H. Chtioui, "On the prescribed scalar curvature problem on the three-dimensional half sphere," Pacific Journal of Mathematics, vol. 221, no. 2, pp. 201-226, 2005.

[4] M. Ben Ayed and M. O. Ahmedou, "On the prescribed scalar curvature on 3-half spheres: multiplicity results and Morse inequalities at infinity," Discrete and Continuous Dynamical Systems A, vol. 23, no. 3, pp. 655-683, 2009.

[5] P. Cherrier, "Problèmes de Neumann non linéaires sur les variétés riemanniennes," Journal of Functional Analysis, vol. 57, no. 2, pp. 154-207, 1984.

[6] Z. Djadli, A. Malchiodi, and M. Ould Ahmedou, "Prescribing scalar and boundary mean curvature on the three dimensional half sphere," The Journal of Geometric Analysis, vol. 13, no. 2, pp. 255-289, 2003.

[7] J. F. Escobar, "Conformal deformation of a Riemannian metric to a scalar flat metric with constant mean curvature on the boundary," Annals of Mathematics, vol. 136, no. 1, pp. 1-50, 1992.

[8] J. F. Escobar, "Conformal metrics with prescribed mean curvature on the boundary," Calculus of Variations and Partial Differential Equations, vol. 4, no. 6, pp. 559-592, 1996.

[9] Z.-C. Han and Y. Li, "The Yamabe problem on manifolds with boundary: existence and compactness results," Duke Mathematical Journal, vol. 99, no. 3, pp. 489-542, 1999.

[10] Z.-C. Han and Y. Li, "The existence of conformal metrics with constant scalar curvature and constant boundary mean curvature," Communications in Analysis and Geometry, vol. 8, no. 4, pp. 809-869, 2000.

[11] Y. Li, "The Nirenberg problem in a domain with boundary," Topological Methods in Nonlinear Analysis, vol. 6, no. 2, pp. 309329, 1995.

[12] R. Yacoub, "Topological arguments in prescribing the scalar curvature under minimal boundary mean curvature condition on $S_{+}^{n}$," Differential and Integral Equations, vol. 21, no. 5-6, pp. 459-476, 2008. 
[13] G. Bianchi and X.-B. Pan, "Yamabe equations on half-spaces," Nonlinear Analysis: Theory, Methods \& Applications A, vol. 37, no. 2, pp. 161-186, 1999.

[14] A. Bahri, Critical Point at Infinity in Some Variational Problems, vol. 182 of Pitman Research Notes in Mathematics Series, Longman Scientific \& Technical, Harlow, UK, 1989.

[15] R. Schoen and D. Zhang, "Prescribed scalar curvature on the $n$-sphere," Calculus of Variations and Partial Differential Equations, vol. 4, no. 1, pp. 1-25, 1996.

[16] H. Brezis and J.-M. Coron, "Convergence of solutions of $H$ systems or how to blow bubbles," Archive for Rational Mechanics and Analysis, vol. 89, no. 1, pp. 21-56, 1985.

[17] P.-L. Lions, "The concentration-compactness principle in the calculus of variations. The limit case. II," Revista Matemática Iberoamericana, vol. 1, no. 2, pp. 45-121, 1985.

[18] M. Struwe, "A global compactness result for elliptic boundary value problems involving limiting nonlinearities," Mathematische Zeitschrift, vol. 187, no. 4, pp. 511-517, 1984.

[19] A. Bahri, "An invariant for Yamabe-type flows with applications to scalar-curvature problems in high dimension," Duke Mathematical Journal, vol. 81, no. 2, pp. 323-466, 1996, A celebration of John F. Nash, Jr.

[20] A. Bahri and J.-M. Coron, "The scalar-curvature problem on the standard three-dimensional sphere," Journal of Functional Analysis, vol. 95, no. 1, pp. 106-172, 1991.

[21] O. Rey, "The role of the Green's function in a nonlinear elliptic equation involving the critical Sobolev exponent," Journal of Functional Analysis, vol. 89, no. 1, pp. 1-52, 1990.

[22] A. Bahri and P. H. Rabinowitz, "Periodic solutions of Hamiltonian systems of three-body type," Annales de l'Institut Henri Poincaré. Analyse Non Linéaire, vol. 8, no. 6, pp. 561-649, 1991. 


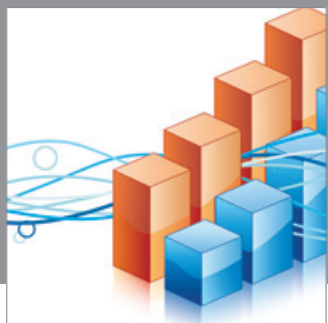

Advances in

Operations Research

mansans

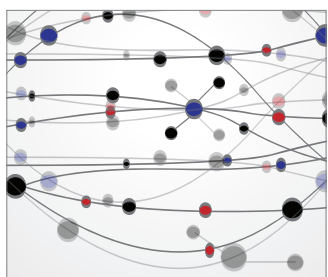

The Scientific World Journal
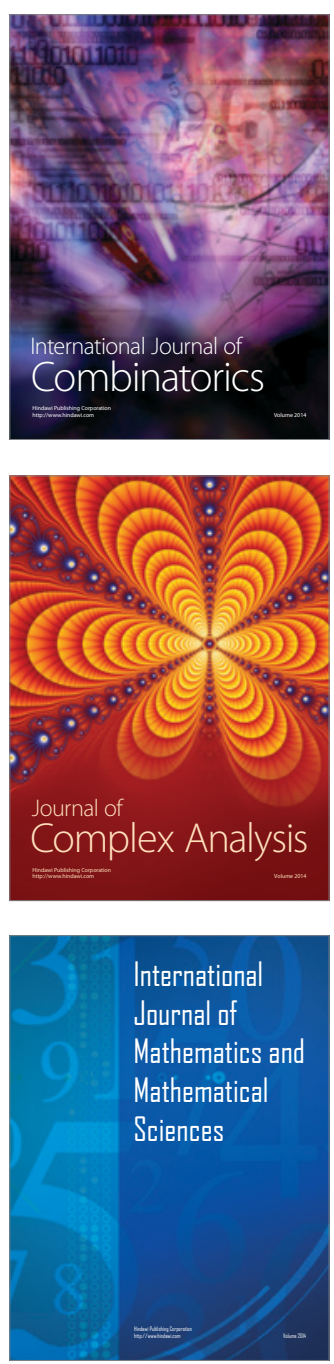
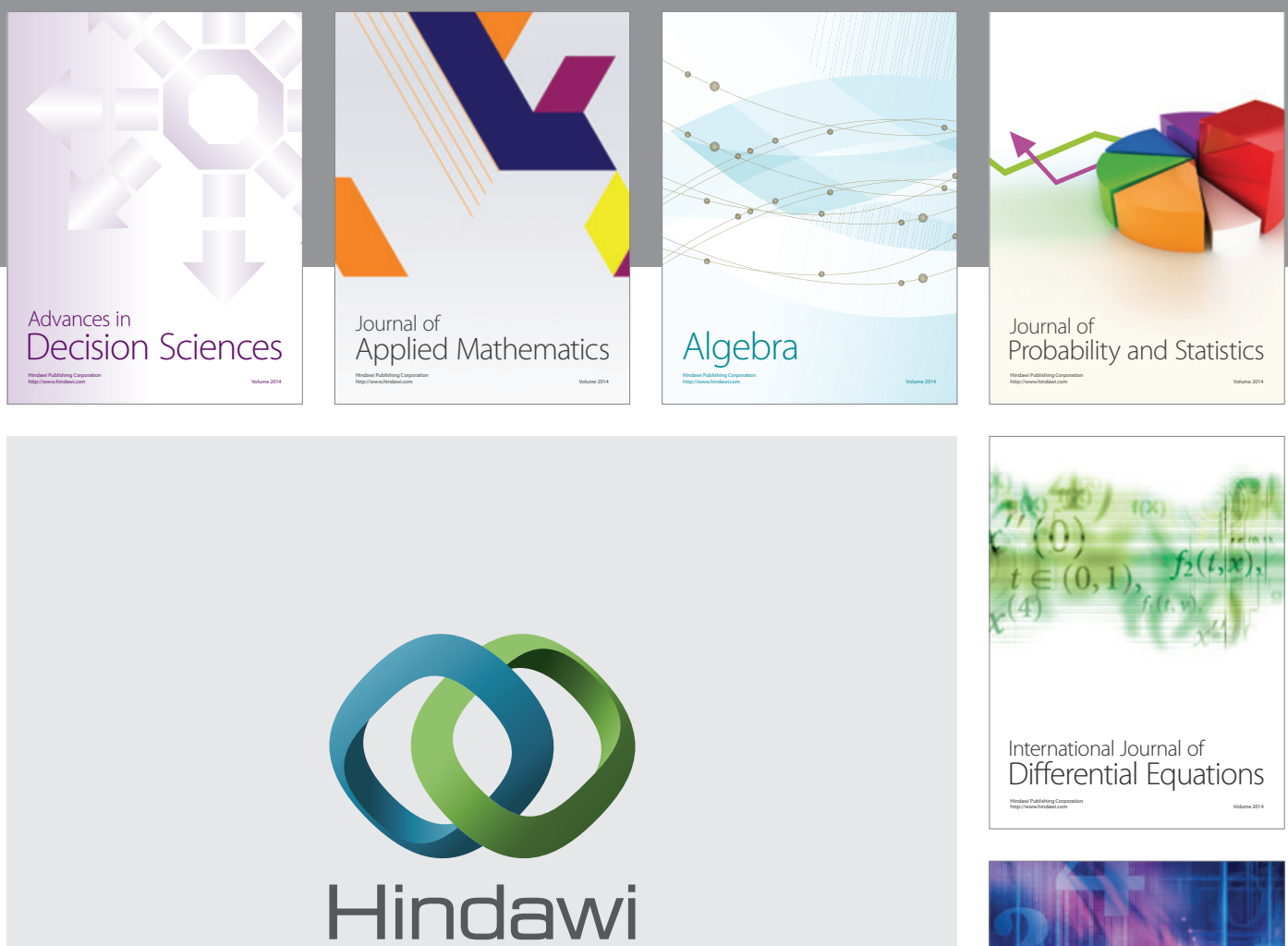

Submit your manuscripts at http://www.hindawi.com
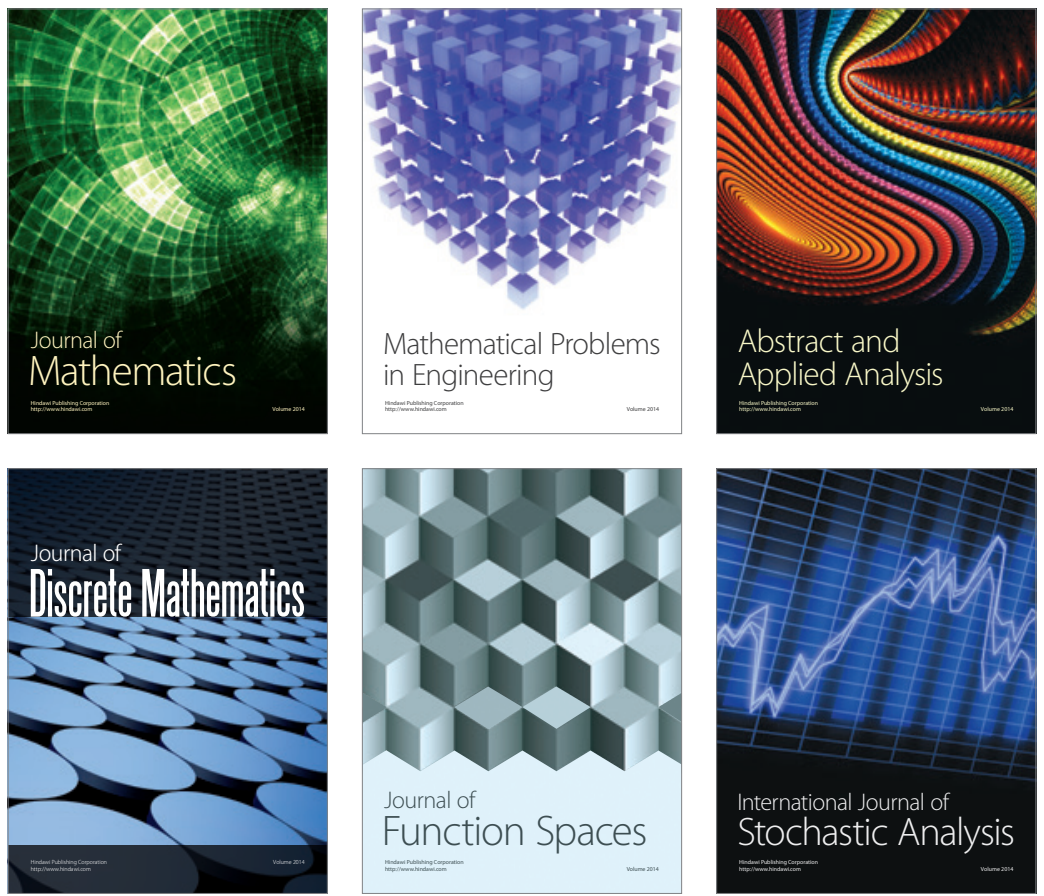

Journal of

Function Spaces

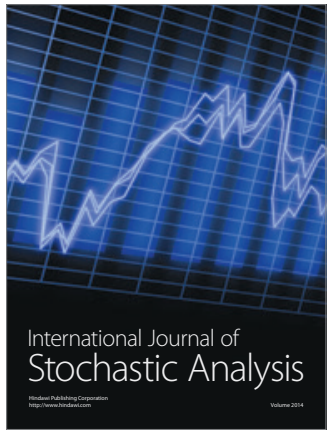

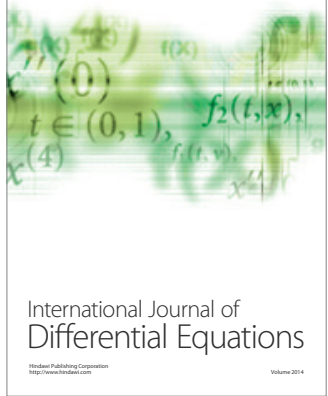
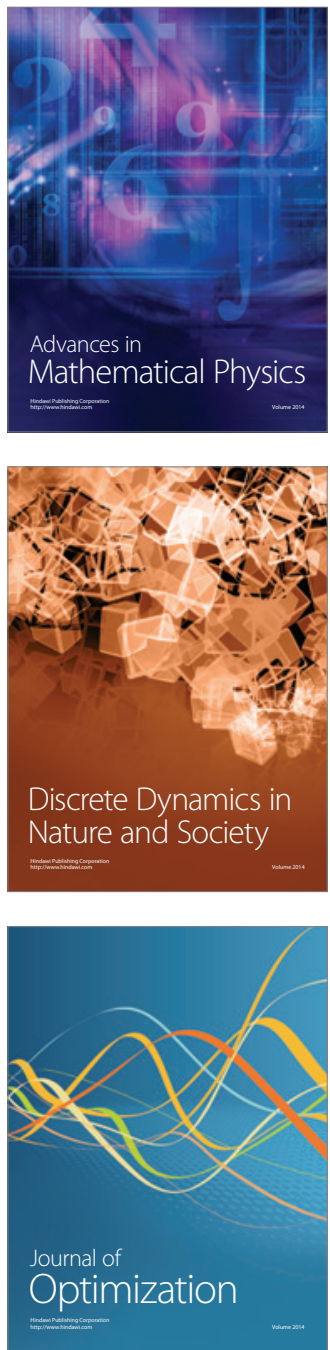